\title{
Sexual Activity in Adolescents and Young Adults through COVID-19 Pandemic
}

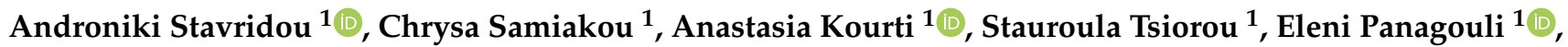 \\ Athanasios Thirios ${ }^{1}$, Theodora Psaltopoulou ${ }^{2}$, Theodoros N. Sergentanis ${ }^{1,2,+} \mathbb{D}$ and Artemis Tsitsika ${ }^{1, *,+}$
}

1 2nd Department of Pediatrics, “P. \& A. Kyriakou” Children's Hospital, School of Medicine, National and Kapodistrian University of Athens, 11527 Athens, Greece; stavroniki@hotmail.com (A.S.); samichry@yahoo.com (C.S.); anastasiakourti.ak@gmail.com (A.K.); st.tsiorou@gmail.com (S.T.); eleni72000@yahoo.gr (E.P.); dunapower@gmail.com (A.T.); tsergentanis@yahoo.gr (T.N.S.)

2 Department of Clinical Therapeutics, "Alexandra" Hospital, School of Medicine, National and Kapodistrian University of Athens, 11528 Athens, Greece; tpsaltop@med.uoa.gr

* Correspondence: info@youth-health.gr; Tel./Fax: +30-2107710824

+ These authors contributed equally to this manuscript.

Citation: Stavridou, A.; Samiakou, C.; Kourti, A.; Tsiorou, S.; Panagouli, E.; Thirios, A.; Psaltopoulou, T.; Sergentanis, T.N.; Tsitsika, A. Sexual Activity in Adolescents and Young Adults through COVID-19 Pandemic. Children 2021, 8, 577. https:// doi.org/10.3390/children8070577

Academic Editor: Brian Littlechild

Received: 16 May 2021

Accepted: 3 July 2021

Published: 5 July 2021

Publisher's Note: MDPI stays neutral with regard to jurisdictional claims in published maps and institutional affiliations.

Copyright: (c) 2021 by the authors. Licensee MDPI, Basel, Switzerland. This article is an open access article distributed under the terms and conditions of the Creative Commons Attribution (CC BY) license (https:// creativecommons.org/licenses/by/ $4.0 /)$.

\begin{abstract}
During the COVID-19 pandemic, it has been postulated that the sexual life of adolescent and young adults has been impacted in various aspects, potentially affecting their well-being. Our aim is to investigate the potential changes in the sexual activity and relationships of adolescents and young adults during the COVID-19 pandemic. In general, a decrease in sexual desire was reported during the COVID-19 pandemic, in both genders. Fewer sexual intercourses and bonding behaviors between partners were associated with loneliness and depressive symptoms. On the contrary, an increase in sexual desire was expressed in a few people, with masturbation to be the most preferable means of satisfaction. The present paper highlights the multifaceted impact of COVID-19 upon the sexual life of adolescents and young adults during the ongoing pandemic. The changes observed in their sexual activity and relationships, could provide the basis of future preventive and educational programs.
\end{abstract}

Keywords: COVID-19; sexual health; adolescents; young adults

\section{Introduction}

In December 2019, the first cases of COVID-19 were reported in Wuhan, China, raising concerns due to rapid spreading. The severe acute respiratory syndrome coronavirus 2 (SARS-CoV-2) is the underlying cause of the coronavirus disease (COVID-19), which has spread worldwide, forcing the World Health Organization (WHO) to declare it as a pandemic on 11 March 2020 [1,2]. Many countries imposed restrictive measures, such as lockdowns, social distancing, wearing masks, and frequent washing of hands through the day [3].

The fear of contacting the virus, as well as the public health measures, led people to diminish their visits to crowded places and thus many have faced difficulties in personal relationships, especially adolescents and young adults [4-6]. The lack of peer-to-peer contacts, due to home confinement and school closures, changed many aspects of life for youths. As a result, sexual activity, intimate relationships, access to contraception, protection from HIV, or other sexually transmitted infections (STIs) and physical, mental, or emotional well-being were adversely affected $[7,8]$. The social distancing that was imposed consequently led to the restriction of contacts between individuals, and therefore has affected the frequency of sexual activity, number of sexual partners, sexual desire and satisfaction, and the use of pornographic content [9]. According to the available literature, all these consequences seem to concern mainly younger individuals, such as adolescents and young adults [4-9]. 
The sexual life and activity of the above-mentioned age groups plays an important role in forming healthy sexual relationships and expressing their sexuality $[8,9]$. Sexuality constitutes a meaningful aspect of human development. The impact of COVID-19 in the sexual life of adolescents and young adults may significantly affect their sexuality. Thorough study of those effects will highlight the needs of youths during the pandemic and will provide substantial information in order to maintain their well-being. Thus, the purpose of this paper was to investigate the potential impacts and changes in the sexual activity and relationships of adolescents and young adults caused by the measures imposed due to the ongoing COVID-19 pandemic and the potential consequences to their sexuality in general.

\section{Methods}

A research of the literature was conducted in the following databases: Google Scholar, PsycInfo, PubMed, SCOPUS, and ERIC up to 14 June 2021. The algorithm used included: (sexual OR sex OR sexuality OR intimate) AND (health OR wellness OR life OR wellbeing OR well-being) AND (teens OR teenagers OR youngster OR youngsters OR adolescence OR adolescent OR adolescents OR "young adults" OR "younger adults" OR “Generation Z" OR juvenile OR juveniles OR youthful) AND (COVID-19 OR SARS-CoV-19 OR SARSCoV-2 OR "2019-nCoV" OR "novel coronavirus"). The references of the eligible studies were searched through a snowballing technique, along with the relevant reviews.

The inclusion criteria were the following: Studies that examined changes in the sexual activity and relationships of adolescents and young adults (11-24 years old) during the ongoing COVID-19 pandemic were considered eligible. Studies that incorporated adolescents and young adults in a significant percentage were also included. Sexual activity must refer to sexual desire, frequency of intercourses, number of sexual partners, sexual satisfaction or dissatisfaction, and sexual preferences among adolescents and young adults. Concerning the type of study, case reports, cohort studies, cross-sectional studies, case series, and case-control studies, were included, while no language restrictions were imposed. The selection of studies was conducted by two authors (C.S. and A.S.) who worked independently. The following variables were used to extract data for each study: Title of the article, name of first author and year of publication, region/country where the survey was conducted, language, study period, study design, sample, sample size, age range and selection of sample, ascertainment and/or association with COVID-19 pandemic, outcomes, statistical analysis, and main findings. Quality assessment was performed in order to present accurate results. For this purpose the Newcastle-Ottawa Scale for cross-sectional studies [10] and cohort studies [11] were used.

\section{Results}

\subsection{Selection of Studies}

The research in the database retrieved 20,246 publications, while 1050 of them were duplicates; 16 studies (49,078 individuals) were finally considered eligible following the inclusion criteria imposed as mentioned above [12-27]. The eligible studies included data from various countries, three of them from China [12-14], four from the USA [15-17,25], while two presented data from UK $[18,19]$, three from Italy $[20,21,26]$, the remaining three from various countries, namely, France [22], Poland [23], and Turkey [24], while one included data from various countries [27]. The majority of them were cross-sectional studies $(n=10)$ and the remaining five were cohort studies, and only one study included mixed methods. Eleven studies referred to the COVID-19 pandemic or outbreak in general, whereas five studies referred to lockdowns (Table 1). 
Table 1. Description of studies examining sexual health in adolescents and young adults during the COVID-19 pandemic.

\begin{tabular}{|c|c|c|c|c|c|c|c|c|c|c|c|c|}
\hline $\begin{array}{l}\text { First Author } \\
\text { (Year) }\end{array}$ & Country & Language & $\begin{array}{l}\text { Study } \\
\text { Period }\end{array}$ & $\begin{array}{c}\text { Study } \\
\text { Design }\end{array}$ & Sample & Sample Size & $\begin{array}{c}\text { Age } \\
\text { Range }\end{array}$ & $\begin{array}{l}\text { Selection of } \\
\text { Sample }\end{array}$ & $\begin{array}{l}\text { Ascertainment } \\
\text { and/or } \\
\text { Association } \\
\text { with the } \\
\text { COVID-19 } \\
\text { Pandemic }\end{array}$ & $\begin{array}{c}\text { Outcomes, } \\
\text { Way/Questionnaires } \\
\text { They Were Measured }\end{array}$ & $\begin{array}{l}\text { Statistical } \\
\text { Analysis }\end{array}$ & Main Findings \\
\hline $\begin{array}{l}\text { Li et al. (2020) } \\
\text { [12] }\end{array}$ & China & English & $\begin{array}{l}\text { 1-5 May } \\
2020\end{array}$ & $\begin{array}{c}\text { Cross- } \\
\text { sectional }\end{array}$ & $\begin{array}{l}\text { Users of an } \\
\text { online ques- } \\
\text { tionnaire } \\
\text { survey } \\
\text { platform }\end{array}$ & $\begin{array}{c}967(541 \\
\text { males) }\end{array}$ & $15-35$ & $\begin{array}{l}15-35 \text { years } \\
\text { old, living in } \\
\text { China and } \\
\text { reported } \\
\text { penetrative } \\
\text { sex }\end{array}$ & $\begin{array}{l}\text { COVID-19 } \\
\text { pandemic }\end{array}$ & $\begin{array}{l}\text { Online survey about } \\
\text { sexual and } \\
\text { reproductive health }\end{array}$ & $\begin{array}{l}\text { Logistic } \\
\text { regression } \\
\text { analysis, } \\
\text { Chi-square } \\
\text { test }\end{array}$ & $\begin{array}{c}\text { A total of } 212(22 \%) \text { reported a } \\
\text { decrease in sexual desire, } 396 \\
\text { reported }(41 \%) \text { a decrease in } \\
\text { the frequency of sex, } 192 \\
\text { reported }(20 \%) \text { a recent } \\
\text { decrease in alcohol } \\
\text { consumption before or during } \\
\text { sexual activities, and } 94(10 \%) \\
\text { reported a decrease in risky } \\
\text { sexual behavior. A total of } 298 \\
(31 \%) \text { reported partner } \\
\text { relationship deterioration } \\
\text { during the pandemic, } 291 \text { ( } 30 \%) \\
\text { reported an increase in } \\
\text { masturbation during the } \\
\text { pandemic, and } 227(23 \%) \\
\text { reported an increase in the use } \\
\text { of pornography. } \\
\text { Accommodations during the } \\
\text { pandemic, exclusive } \\
\text { relationship status, sexual } \\
\text { desire, and sexual satisfaction } \\
\text { were closely related to partner } \\
\text { relationships. }\end{array}$ \\
\hline $\begin{array}{l}\text { Li W. et al. } \\
\text { (2020) } \\
\text { [13] }\end{array}$ & China & English & $\begin{array}{c}\text { 13-15 March } \\
2020\end{array}$ & $\begin{array}{c}\text { Cross- } \\
\text { sectional }\end{array}$ & $\begin{array}{l}\text { Users of } \\
\text { Han Chinese } \\
\text { ethnicity of } \\
\text { social media } \\
\text { platforms }\end{array}$ & $\begin{array}{c}459(270 \\
\text { males })\end{array}$ & $18-45$ & $\begin{array}{l}\text { Participants } \\
\text { aged } \\
18-45 \text { years, } \\
\text { with a } \\
\text { history of } \\
\text { sexual } \\
\text { activity }\end{array}$ & $\begin{array}{l}\text { COVID-19 } \\
\text { pandemic }\end{array}$ & $\begin{array}{l}\text { 12-item online } \\
\text { questionnaire } \\
\text { assessing present and } \\
\text { previous sexual } \\
\text { behaviors }\end{array}$ & $\begin{array}{l}\text { Descriptive } \\
\text { statistics and } \\
\text { logistic } \\
\text { regression } \\
\text { analysis }\end{array}$ & $\begin{array}{l}\text { A reduction in sexual desire } \\
\text { was experienced ( } 25 \%) \text { while } \\
44 \% \text { reported a decrease in the } \\
\text { number of sexual partners, } \\
\text { with men reporting more }(53 \% \\
\text { vs. } 30 \%) \text {. Lower frequency in } \\
\text { sexual intercourse was } \\
\text { reported by } 37 \% \text {, including } 26 \% \\
\text { married men and } 28 \% \text { married } \\
\text { women. From married men } \\
(49 \%) \text { and married women } \\
(29 \%) \text { a decrease in the number } \\
\text { of sexual partners was reported. } \\
\text { A total of } 32 \% \text { of men and } 39 \% \\
\text { of women experienced a } \\
\text { reduction in sexual satisfaction } \\
\text { and in risky sexual behaviors. } \\
\text { Only } 18 \% \text { of men and } 8 \% \text { of } \\
\text { women experienced increased } \\
\text { sexual desire. }\end{array}$ \\
\hline
\end{tabular}


Table 1. Cont.

\begin{tabular}{|c|c|c|c|c|c|c|c|c|c|c|c|c|}
\hline $\begin{array}{l}\text { First Author } \\
\text { (Year) }\end{array}$ & Country & Language & $\begin{array}{l}\text { Study } \\
\text { Period }\end{array}$ & $\begin{array}{l}\text { Study } \\
\text { Design }\end{array}$ & Sample & Sample Size & $\begin{array}{c}\text { Age } \\
\text { Range }\end{array}$ & $\begin{array}{l}\text { Selection of } \\
\text { Sample }\end{array}$ & $\begin{array}{c}\text { Ascertainment } \\
\text { and/or } \\
\text { Association } \\
\text { with the } \\
\text { COVID-19 } \\
\text { Pandemic }\end{array}$ & $\begin{array}{c}\text { Outcomes, } \\
\text { Way/Questionnaires } \\
\text { They Were Measured }\end{array}$ & $\begin{array}{l}\text { Statistical } \\
\text { Analysis }\end{array}$ & Main Findings \\
\hline $\begin{array}{l}\text { Landry S. et al. } \\
\text { (2020) } \\
{[22]}\end{array}$ & France & French & $\begin{array}{l}\text { 7th and the } \\
\text { 8th week of } \\
\text { the first } \\
\text { lockdown in } \\
\text { France }\end{array}$ & Cohort & $\begin{array}{l}\text { Users of } \\
\text { LinkedIn, } \\
\text { Facebook, } \\
\text { and Twitter }\end{array}$ & $\begin{array}{c}844(290 \\
\text { males) } \\
\text { (couples } n= \\
\text { of } 437,143 \\
\text { are males), } \\
\text { (singles }= \\
\text { out of } 407, \\
147 \text { are } \\
\text { males) }\end{array}$ & $\begin{array}{c}15- \\
70 \text { years } \\
\text { old. } 71.2 \% \\
\text { of self- } \\
\text { confined } \\
\text { couples } \\
\text { were } \\
15-30- \\
\text { year-olds } \\
\text { and } 88.2 \% \\
\text { of singles } \\
\text { were } \\
15-30- \\
\text { years-old. }\end{array}$ & $\begin{array}{c}\text { Participants } \\
\text { aged 15-70 } \\
\text { years, with a } \\
\text { history of } \\
\text { sexual } \\
\text { activity }\end{array}$ & Lockdown & $\begin{array}{l}\text { Two questionnaires, } \\
\text { one for participants' } \\
\text { confining themselves } \\
\text { in couple, one for } \\
\text { participants' } \\
\text { confining themselves } \\
\text { as singles, assessing } \\
\text { the demographics and } \\
\text { comparing present } \\
\text { (during lockdown) } \\
\text { with previous sexual } \\
\text { behaviors }\end{array}$ & $\begin{array}{l}\text { Descriptive } \\
\text { statistics and } \\
\text { frequency } \\
\text { measurements }\end{array}$ & $\begin{array}{l}\text { Changes in sexual habits were } \\
\text { reported in both couples and } \\
\text { those without partners during } \\
\text { this period. A decrease in } \\
\text { sexual desire was reported in } \\
10.4 \% \text { of females and } 9 \% \text { of } \\
\text { males. }\end{array}$ \\
\hline $\begin{array}{l}\text { Hensel D. et al. } \\
\text { (2020) } \\
{[15]}\end{array}$ & USA & English & $\begin{array}{c}\text { 10-20 April } \\
2020\end{array}$ & $\begin{array}{c}\text { Cross- } \\
\text { sectional }\end{array}$ & $\begin{array}{l}\text { Users of a } \\
\text { web research } \\
\text { panel }\end{array}$ & $\begin{array}{c}1010(48.5 \% \\
\text { males) }\end{array}$ & $\begin{array}{l}18-94 \text { (a } \\
\text { significant } \\
\text { percent- } \\
\text { age 38.3\% } \\
\text { in ages } \\
18-39)\end{array}$ & $\begin{array}{l}\text { Representative } \\
\text { of the } \\
\text { overall US } \\
\text { population }\end{array}$ & $\begin{array}{l}\text { COVID-19 } \\
\text { pandemic }\end{array}$ & $\begin{array}{l}\text { The 26-item online } \\
\text { survey assessing } \\
\text { demographics, } \\
\text { especially the } \\
\text { predictor children in } \\
\text { home and } \\
\text { self-reported past } \\
\text { month changes in } \\
10 \text { solo and partnered } \\
\text { sexual behavior } \\
\text { categories. Five items } \\
\text { for the past month of } \\
\text { depressive symptoms } \\
\text { and three items for } \\
\text { loneliness. }\end{array}$ & $\begin{array}{l}\text { Multinomial } \\
\text { logistic } \\
\text { regression }\end{array}$ & $\begin{array}{l}\text { In the past month of sexual } \\
\text { behavior, half of all adults } \\
\text { reported change, mostly a } \\
\text { decrease. The younger the age } \\
\text { of their children (under the age } \\
\text { of five, the greater the } \\
\text { likelihood of stability and/or } \\
\text { increased partnered behaviors, } \\
\text { whereas having } \\
\text { elementary-aged children was } \\
\text { often associated with a } \\
\text { decrease in these behaviors. } \\
\text { Past month of depressive } \\
\text { symptoms and loneliness were } \\
\text { associated with both reduced } \\
\text { partnered bonding behaviors } \\
\text { and partnered sexual behaviors } \\
\text { The greater the perceived risk } \\
\text { of COVID-19, the lesser the } \\
\text { reported solo and partnered } \\
\text { sexual behaviors. Stability in } \\
\text { partnered sexual behaviors was } \\
\text { reported in people with greater } \\
\text { COVID-19 knowledge. }\end{array}$ \\
\hline
\end{tabular}


Table 1. Cont.

\begin{tabular}{|c|c|c|c|c|c|c|c|c|c|c|c|c|}
\hline $\begin{array}{l}\text { First Author } \\
\text { (Year) }\end{array}$ & Country & Language & $\begin{array}{l}\text { Study } \\
\text { Period }\end{array}$ & $\begin{array}{l}\text { Study } \\
\text { Design }\end{array}$ & Sample & Sample Size & $\begin{array}{c}\text { Age } \\
\text { Range }\end{array}$ & $\begin{array}{l}\text { Selection of } \\
\text { Sample }\end{array}$ & $\begin{array}{l}\text { Ascertainment } \\
\text { and/or } \\
\text { Association } \\
\text { with the } \\
\text { COVID-19 } \\
\text { Pandemic }\end{array}$ & $\begin{array}{c}\text { Outcomes, } \\
\text { Way/Questionnaires } \\
\text { They Were Measured }\end{array}$ & $\begin{array}{l}\text { Statistical } \\
\text { Analysis }\end{array}$ & Main Findings \\
\hline $\begin{array}{l}\text { Luetke, M. } \\
\text { et al. (2020) } \\
{[16]}\end{array}$ & USA & English & $\begin{array}{c}10-20 \text { April } \\
2020\end{array}$ & $\begin{array}{c}\text { Cross- } \\
\text { sectional }\end{array}$ & $\begin{array}{l}\text { Users of a } \\
\text { web research } \\
\text { panel }\end{array}$ & $\begin{array}{c}742(49 \% \\
\text { males) }\end{array}$ & $\begin{array}{l}18-94(\mathrm{a} \\
\text { significant } \\
\text { percent- } \\
\text { age 36.7\% } \\
\text { in ages } \\
18-39)\end{array}$ & $\begin{array}{l}\text { Representative } \\
\text { of the } \\
\text { overall U.S. } \\
\text { population }\end{array}$ & $\begin{array}{l}\text { COVID-19 } \\
\text { pandemic }\end{array}$ & $\begin{array}{l}\text { The 26-item online } \\
\text { survey assessing } \\
\text { demographics and } \\
\text { self-reported past } \\
\text { month changes in } \\
10 \text { solo and partnered } \\
\text { sexual behavior } \\
\text { categories. Five items } \\
\text { for the past month of } \\
\text { depressive symptoms } \\
\text { and three items for } \\
\text { loneliness. Two other } \\
\text { secondary outcomes } \\
\text { were assessed: } \\
\text { Orgasm and } \\
\text { emotional closeness at } \\
\text { last sexual event }\end{array}$ & $\begin{array}{l}\text { Chi-square } \\
\text { tests, student's } \\
t \text {-tests, and } \\
\text { multinomial } \\
\text { logistic } \\
\text { regression }\end{array}$ & $\begin{array}{l}\text { A decreased frequency in } \\
\text { several intimate and sexual } \\
\text { behaviors was associated with } \\
\text { conflict, which also tended to } \\
\text { be associated with lower levels } \\
\text { of self-reported orgasms and } \\
\text { emotional connectedness at } \\
\text { participants' most recent past } \\
\text { month sexual events. The } \\
\text { association between conflict } \\
\text { and decreased frequency of the } \\
\text { intimate and sexual behaviors } \\
\text { tended to be stronger among } \\
\text { men than among women. } \\
\text { Greater difficulties were } \\
\text { reported in romantic and/or } \\
\text { sexual relationships. }\end{array}$ \\
\hline $\begin{array}{l}\text { Sanchez T. } \\
\text { et al. }(2020) \\
{[17]}\end{array}$ & USA & English & $\begin{array}{c}\text { 2-13 April } \\
2020\end{array}$ & $\begin{array}{c}\text { Cross- } \\
\text { sectional }\end{array}$ & $\begin{array}{l}\text { Participants } \\
\text { of the } \\
\text { American } \\
\text { Men's } \\
\text { Internet } \\
\text { Survey } \\
\text { (AMIS) who } \\
\text { conducted } \\
\text { mailed } \\
\text { HIV/STI } \\
\text { testing the } \\
\text { past } 2 \text { years }\end{array}$ & 1051 & $15-40+$ & $\begin{array}{c}15 \text { years or } \\
\text { older, male } \\
\text { sex at birth, } \\
\text { resided in } \\
\text { the US, and } \\
\text { reported } \\
\text { oral or anal } \\
\text { sex with a } \\
\text { man at least } \\
\text { once at any } \\
\text { time in the } \\
\text { past }\end{array}$ & $\begin{array}{l}\text { COVID-19 } \\
\text { pandemic }\end{array}$ & Online questionnaire & $\begin{array}{l}\text { Differences in } \\
\text { the prevalence } \\
\text { of adverse } \\
\text { COVID-19- } \\
\text { related impacts } \\
\text { by participant } \\
\text { age were } \\
\text { reported as } \\
\text { bivariate } \\
\text { prevalence } \\
\text { ratios (PR) and } \\
95 \% \\
\text { confidence } \\
\text { intervals (CI) }\end{array}$ & $\begin{array}{l}\text { The majority of the participants } \\
\text { reported fewer sex partners } \\
\text { and opportunities to have sex } \\
\text { due to COVID-19 and a } \\
\text { decrease or unchanged usage } \\
\text { of dating or hook-up apps, } \\
\text { mainly to connect with other } \\
\text { men. Condom access and } \\
\text { usage were unchanged due to } \\
\text { COVID-19. One-tenth of } \\
\text { participants had increased use } \\
\text { of recreational drugs and } \\
\text { one-quarter had increased } \\
\text { alcohol consumption. }\end{array}$ \\
\hline $\begin{array}{l}\text { Thomson- } \\
\text { Glover R. et al. } \\
\text { (2020) } \\
{[18]}\end{array}$ & UK & English & $\begin{array}{c}10 \text { February } \\
\text { to } 22 \text { March } \\
2020 \text { (pre- } \\
\text { lockdown) } \\
\text { AND 23 } \\
\text { March to } 30 \\
\text { April 2020 } \\
\text { (during } \\
\text { lockdown) }\end{array}$ & Cohort & $\begin{array}{l}\text { Young } \\
\text { people }\end{array}$ & $\begin{array}{c}25,651 \\
(24,910 \\
\text { attendances } \\
\text { for sexual } \\
\text { health } \\
\text { services and } \\
741 \\
\text { emergency } \\
\text { contracep- } \\
\text { tion } \\
\text { attendances) }\end{array}$ & $\begin{array}{l}17 \text { and } \\
\text { under and } \\
18 \text { and } \\
\text { over }\end{array}$ & $\begin{array}{l}\text { Sexual } \\
\text { health } \\
\text { attendances } \\
\text { among } \\
\text { young } \\
\text { people (YP) } \\
\text { within a } \\
\text { semirural } \\
\text { service } \\
\text { setting and } \\
\text { at services } \\
\text { based in } \\
\text { London and } \\
\text { Surrey }\end{array}$ & Lockdown & Hospital records & NR & $\begin{array}{c}\text { Due to COVID-19 restrictions, } \\
\text { reduction in demand for sexual } \\
\text { health services (SHS) may } \\
\text { reflect reduced sexual activity } \\
\text { and partnerships among young } \\
\text { people. }\end{array}$ \\
\hline
\end{tabular}


Table 1. Cont.

\begin{tabular}{|c|c|c|c|c|c|c|c|c|c|c|c|c|}
\hline $\begin{array}{l}\text { First Author } \\
\text { (Year) }\end{array}$ & Country & Language & $\begin{array}{l}\text { Study } \\
\text { Period }\end{array}$ & $\begin{array}{c}\text { Study } \\
\text { Design }\end{array}$ & Sample & Sample Size & $\begin{array}{c}\text { Age } \\
\text { Range }\end{array}$ & $\begin{array}{l}\text { Selection of } \\
\text { Sample }\end{array}$ & $\begin{array}{c}\text { Ascertainment } \\
\text { and/or } \\
\text { Association } \\
\text { with the } \\
\text { COVID-19 } \\
\text { Pandemic }\end{array}$ & $\begin{array}{c}\text { Outcomes, } \\
\text { Way/Questionnaires } \\
\text { They Were Measured }\end{array}$ & $\begin{array}{l}\text { Statistical } \\
\text { Analysis }\end{array}$ & Main Findings \\
\hline $\begin{array}{c}\text { Jianjun et al. } \\
\text { (2020) } \\
{[14]}\end{array}$ & China & English & NR & $\begin{array}{c}\text { Cross- } \\
\text { sectional }\end{array}$ & $\begin{array}{l}\text { Undergraduate } \\
\text { students }\end{array}$ & $\begin{array}{c}3219 \\
\text { (714 males) }\end{array}$ & $17-24$ & $\begin{array}{l}\text { Recruited } \\
\text { online via } \\
\text { smartphone } \\
\text { from } \\
\text { universities }\end{array}$ & $\begin{array}{l}\text { COVID-19 } \\
\text { outbreak }\end{array}$ & $\begin{array}{c}\text { Adolescent } \\
\text { Self-Rating } \\
\text { Life-Events Check } \\
\text { List (ASLEC), and } \\
8 \text { psychological } \\
\text { behaviors indicators } \\
\text { assessed individual } \\
\text { subjective emotion for } \\
\text { the COVID-19 } \\
\text { stress-Items, } \\
\text { Perceived Social } \\
\text { Support Scale } \\
\text { (PSSS)-Sexual } \\
\text { Compulsivity Scale } \\
\text { (SCS) }\end{array}$ & $\begin{array}{l}\text { SPSS } 22.0 \text { and } \\
\text { MPLUS 7.0- } \\
\text { differences in } \\
\text { testing, } \\
\text { descriptive } \\
\text { statistics, } \\
\text { correlation } \\
\text { analyses, } \\
\text { hierarchical } \\
\text { regressing } \\
\text { analyses, and } \\
\text { the testing of } \\
\text { mediator } \\
\text { (moderator) } \\
\text { model }\end{array}$ & $\begin{array}{l}\text { Females reported higher } \\
\text { COVID-19-related stress, PSSS, } \\
\text { and SCS than males. In } \\
\text { particular, COVID-19 stress } \\
(p<0.001) \text { and PSSS }(p<0.01) \\
\text { was significant. Participants } \\
\text { over } 21 \text { had lower } \\
\text { COVID-19-related stress, and } \\
\text { higher PSSS and SCS compared } \\
\text { to younger people, with the } \\
\text { differences of COVID-19 } \\
\text { stressing }(p<0.001) \text { being } \\
\text { significant. Urban participants } \\
\text { had higher COVID-19 stressing, } \\
\text { and lower PSSS and SCS } \\
\text { compared to rural participants, } \\
\text { while only COVID-19-related } \\
\text { stress }(p<0.001) \text { and SCS } \\
\text { ( } p<0.05) \text { differences were } \\
\text { significant. Therefore, these } \\
\text { results showed that differences } \\
\text { in COVID-19 stress, PSSS, and } \\
\text { SCS were significant for gender, } \\
\text { years, and urban/rural status. }\end{array}$ \\
\hline
\end{tabular}


Table 1. Cont.

\begin{tabular}{|c|c|c|c|c|c|c|c|c|c|c|c|c|}
\hline $\begin{array}{l}\text { First Author } \\
\text { (Year) }\end{array}$ & Country & Language & $\begin{array}{l}\text { Study } \\
\text { Period }\end{array}$ & $\begin{array}{c}\text { Study } \\
\text { Design }\end{array}$ & Sample & Sample Size & $\begin{array}{c}\text { Age } \\
\text { Range }\end{array}$ & $\begin{array}{l}\text { Selection of } \\
\text { Sample }\end{array}$ & $\begin{array}{c}\text { Ascertainment } \\
\text { and/or } \\
\text { Association } \\
\text { with the } \\
\text { COVID-19 } \\
\text { Pandemic } \\
\end{array}$ & $\begin{array}{c}\text { Outcomes, } \\
\text { Way/Questionnaires } \\
\text { They Were Measured }\end{array}$ & $\begin{array}{l}\text { Statistical } \\
\text { Analysis }\end{array}$ & Main Findings \\
\hline $\begin{array}{l}\text { Cocci A. et al. } \\
\text { (2020) } \\
{[20]}\end{array}$ & Italy & English & $\begin{array}{c}\text { March to } \\
\text { April } 2020\end{array}$ & $\begin{array}{c}\text { Cross- } \\
\text { sectional }\end{array}$ & $\begin{array}{c}\text { From social } \\
\text { networks } \\
\text { (Instagram } \\
\text { and } \\
\text { Facebook) }\end{array}$ & 1515 & $\begin{array}{l}\text { Mean age } \\
\quad=21\end{array}$ & $\begin{array}{c}\text { Posts on } \\
\text { social } \\
\text { networks } \\
\text { (Instagram } \\
\text { and } \\
\text { Facebook), } \\
\text { through } \\
\text { Google } \\
\text { Forms }\end{array}$ & Lockdown & $\begin{array}{l}\text { The Beck Depression } \\
\text { Inventory (BDI-II) } \\
\text { and the Beck Anxiety } \\
\text { Inventory (BAI) in the } \\
\text { Italian validated } \\
\text { version, sexual } \\
\text { satisfaction was } \\
\text { evaluated by asking } \\
\text { the question "Overall, } \\
\text { how satisfied are you } \\
\text { with your sex life } \\
\text { during quarantine on } \\
\text { a scale ranging from } 1 \\
\text { (not at all satisfied) to } \\
5 \text { (extremely } \\
\text { satisfied)?" }\end{array}$ & $\begin{array}{l}\text { Multivariate } \\
\text { logistic } \\
\text { regression } \\
\text { analysis, BDI } \\
\text { score, BAI } \\
\text { score, } \\
\text { Chi-square test }\end{array}$ & $\begin{array}{c}\text { Median BDI score was 10.0 } \\
\text { (IQR: } 5.0-16.0 \text { ) and median BAI } \\
\text { score was 11.0 (IQR: 6.0-20.0). } \\
\text { A total of 602 ( } 39.74 \% \text { ) } \\
\text { respondents answered that } \\
\text { quarantine increased } \\
\text { autoerotism more than before. } \\
\text { Although only } 7.46 \% \text { of the } \\
\text { participants reported "no } \\
\text { satisfaction" before the } \\
\text { quarantine, } 53.53 \% \text { complained } \\
\text { about that during quarantine } \\
\text { (p<0.01). Women had greater } \\
\text { depression (BDI-male: } 8.0 \text { [IQR } \\
4.0-13.0] \text { ] BDI-female: } 11.0 \\
\text { [IQR 6.0-17.0]; } p<0.01 \text { ) and } \\
\text { anxiety versus males } \\
\text { (BAI-male: } 7.0 \text { [IQR 3.0-14.0]; } \\
\text { BAI-female 13.0 [IQR 7.0-23.0]; } \\
p<0.01 \text { ). Multivariate logistic } \\
\text { regression analysis, presented } \\
\text { that age (odds ratio [OR]: } 0.96 ; \\
p<0.01 \text { ) and BDI (OR: } 1.07 ; \\
p<0.01 \text { in males and age (OR: } \\
0.96 ; p<0.01 \text { ), BDI (OR: } 1.03 ; \\
p<0.01 \text { ) and "knowing people } \\
\text { positive at COVID" (OR: } 0.78 ; \\
0.05 \text { in women were } \\
\text { significant predictors of sexual } \\
\text { dissatisfaction. }\end{array}$ \\
\hline $\begin{array}{l}\text { Panzeri M. } \\
\text { et al. (2020) } \\
\text { [21] }\end{array}$ & Italy & English & $\begin{array}{c}11 \text { April to } 5 \\
\text { May } 2020\end{array}$ & $\begin{array}{c}\text { Cross- } \\
\text { sectional }\end{array}$ & $\begin{array}{c}\text { Cohabiting } \\
\text { couples }\end{array}$ & $\begin{array}{c}124(26.6 \% \\
\text { males) }\end{array}$ & $\begin{array}{c}23-60 \\
(61.5 \% \\
\text { under } 34)\end{array}$ & $\begin{array}{l}\text { Online } \\
\text { survey }\end{array}$ & Lockdown & $\begin{array}{c}\text { Online questionnaire } \\
\text { including Quality of } \\
\text { Marriage Index } \\
\text { (QMI)-Two factors } \\
\text { of the Sexual Desire } \\
\text { Inventory } \\
\text { (SDI)-Three factors } \\
\text { of the Depression } \\
\text { Anxiety Stress } \\
\text { Scales-21 } \\
\text { (DASS-21)-The total } \\
\text { score of the Patient } \\
\text { Health Questionnaire } \\
\text { (PHQ-15) }\end{array}$ & $\begin{array}{l}\text { Logistic } \\
\text { regression } \\
\text { analysis, } t \text {-test } \\
\text { analysis, and } \\
\text { Chi-square } \\
\text { analysis }\end{array}$ & $\begin{array}{l}\text { Most couples responded that } \\
\text { they did not perceive any } \\
\text { differences in their sexuality, } \\
\text { despite the pandemic's } \\
\text { consequences. Some female } \\
\text { participants reported a } \\
\text { decrease in pleasure, } \\
\text { satisfaction, desire, and arousal. } \\
\text { The main reasons for this } \\
\text { seemed to be worry, lack of } \\
\text { privacy, and stress. Even } \\
\text { though participants seemed to } \\
\text { show high levels of resilience, } \\
\text { the negative aspects of } \\
\text { lockdown could affect their } \\
\text { quality of sexual life. }\end{array}$ \\
\hline
\end{tabular}


Table 1. Cont.

\begin{tabular}{|c|c|c|c|c|c|c|c|c|c|c|c|c|}
\hline $\begin{array}{l}\text { First Author } \\
\text { (Year) }\end{array}$ & Country & Language & $\begin{array}{l}\text { Study } \\
\text { Period }\end{array}$ & $\begin{array}{c}\text { Study } \\
\text { Design }\end{array}$ & Sample & Sample Size & $\begin{array}{c}\text { Age } \\
\text { Range }\end{array}$ & $\begin{array}{l}\text { Selection of } \\
\text { Sample }\end{array}$ & $\begin{array}{c}\text { Ascertainment } \\
\text { and/or } \\
\text { Association } \\
\text { with the } \\
\text { COVID-19 } \\
\text { Pandemic } \\
\end{array}$ & $\begin{array}{c}\text { Outcomes, } \\
\text { Way/Questionnaires } \\
\text { They Were Measured }\end{array}$ & $\begin{array}{l}\text { Statistical } \\
\text { Analysis }\end{array}$ & Main Findings \\
\hline $\begin{array}{c}\text { Yuksel B. \& } \\
\text { Osgor F. (2020) } \\
\text { [24] }\end{array}$ & Turkey & English & $\begin{array}{l}\text { 1 February } \\
2018 \text { to } 30 \\
\text { September } \\
2019 \text { and } 11 \\
\text { March to } 12 \\
\text { April } 2020\end{array}$ & Cohort & $\begin{array}{l}\text { Women } \\
\text { patients in } \\
\text { Haseki } \\
\text { Training and } \\
\text { Research } \\
\text { Hospital and } \\
\text { Esenler } \\
\text { Maternity } \\
\text { and } \\
\text { Children's } \\
\text { Hospital }\end{array}$ & 58 & $\begin{array}{c}\text { Mean age } \\
=27.6\end{array}$ & $\begin{array}{l}\text { Patient data } \\
\text { from the } \\
\text { electronic } \\
\text { data system }\end{array}$ & $\begin{array}{l}\text { COVID-19 } \\
\text { pandemic }\end{array}$ & $\begin{array}{c}\text { Female Sexual } \\
\text { Function Index (FSFI) } \\
\text { questionnaire }\end{array}$ & $\begin{array}{l}\text { The } \\
\text { Kolmogorov- } \\
\text { Smirnov test, } \\
\text { the paired } \\
\text { sample } t \text {-test } \\
\text { and McNemar } \\
\text { test were used } \\
\text { to compare the } \\
\text { averages and } \\
\text { percentages }\end{array}$ & $\begin{array}{l}\text { During the pandemic, the } \\
\text { average frequency of sexual } \\
\text { intercourse was significantly } \\
\text { increased compared with } \\
\text { 6-12 months prior ( } 2.4 \text { vs. 1.9, } \\
p=0.001) \text {. Female Sexual } \\
\text { Function Index (FSFI) scores } \\
\text { were significantly better before } \\
\text { the pandemic compared with } \\
\text { scores during the pandemic } \\
\text { (20.52 vs. } 17.56, p=0.001) .\end{array}$ \\
\hline $\begin{array}{l}\text { Fuchs A. et al. } \\
\text { (2020) } \\
{[23]}\end{array}$ & Poland & English & $\begin{array}{l}\text { March to } \\
\text { April } 2020\end{array}$ & Cohort & $\begin{array}{l}\text { Research in } \\
\text { Hospital and } \\
\text { Esenler } \\
\text { Maternity } \\
\text { and } \\
\text { Children's } \\
\text { Hospital }\end{array}$ & 764 & $\begin{array}{l}\text { Mean age } \\
\quad=25.1\end{array}$ & $\begin{array}{c}\text { Patients of } \\
\text { Department } \\
\text { of } \\
\text { Pregnancy } \\
\text { Pathology, } \\
\text { Department } \\
\text { of Woman's } \\
\text { Health, } \\
\text { School of } \\
\text { Health } \\
\text { Sciences in } \\
\text { Katowice, } \\
\text { Medical } \\
\text { University } \\
\text { of Silesia }\end{array}$ & $\begin{array}{l}\text { COVID-19 } \\
\text { pandemic }\end{array}$ & $\begin{array}{c}\text { Female Sexual } \\
\text { Function Index (FSFI) } \\
\text { questionnaire }\end{array}$ & $\begin{array}{l}\text { Wilcoxon's } \\
\text { rank test, } \\
\text { comparison } \\
\text { Chi22, } \\
\text { Kruskal-Wallis } \\
\text { test, U Mann- } \\
\text { Whitney } \\
\text { test }\end{array}$ & $\begin{array}{c}\text { The overall FSFI score before } \\
\text { the pandemic was } 30.1 \pm 4.4 \\
\text { and during, it changed to } 25.8 \\
\pm 9.7 \text {. Desire, arousal, } \\
\text { lubrication, orgasm, } \\
\text { satisfaction, and pain scores } \\
\text { decreased as well ( } p<0.001) \text {. } \\
\text { There was statistically } \\
\text { significant association between } \\
\text { the workplace and the change } \\
\text { of FSFI scores before and } \\
\text { during the COVID-19 } \\
\text { pandemic }(p<0.01) \text {. The } \\
\text { largest decrease in FSFI score } \\
\text { was noticed in the group of } \\
\text { women who did not work at all } \\
(5.2 \pm 9.9) \text {. }\end{array}$ \\
\hline
\end{tabular}


Table 1. Cont.

\begin{tabular}{|c|c|c|c|c|c|c|c|c|c|c|c|c|}
\hline $\begin{array}{l}\text { First Author } \\
\text { (Year) }\end{array}$ & Country & Language & $\begin{array}{l}\text { Study } \\
\text { Period }\end{array}$ & $\begin{array}{c}\text { Study } \\
\text { Design }\end{array}$ & Sample & Sample Size & $\begin{array}{c}\text { Age } \\
\text { Range }\end{array}$ & $\begin{array}{l}\text { Selection of } \\
\text { Sample }\end{array}$ & $\begin{array}{c}\text { Ascertainment } \\
\text { and/or } \\
\text { Association } \\
\text { with the } \\
\text { COVID-19 } \\
\text { Pandemic } \\
\end{array}$ & $\begin{array}{c}\text { Outcomes, } \\
\text { Way/Questionnaires } \\
\text { They Were Measured }\end{array}$ & $\begin{array}{l}\text { Statistical } \\
\text { Analysis }\end{array}$ & Main Findings \\
\hline $\begin{array}{l}\text { Jacob L. et al. } \\
\text { (2020) } \\
{[19]}\end{array}$ & UK & English & $\begin{array}{l}\text { 17 March } \\
2020\end{array}$ & $\begin{array}{c}\text { Cross- } \\
\text { sectional }\end{array}$ & $\begin{array}{l}\text { Adults aged } \\
18 \text { years and } \\
\text { over, } \\
\text { currently } \\
\text { residing in } \\
\text { the UK and } \\
\text { self- } \\
\text { isolating/social- } \\
\text { distancing } \\
\text { due to } \\
\text { COVID-19 }\end{array}$ & $\begin{array}{c}868(\text { male } \\
\quad 36.9 \%) \\
-\quad \\
\end{array}$ & $\begin{array}{l}18-75 \\
(32.4 \% \\
18-34 \\
\text { years })\end{array}$ & $\begin{array}{l}\text { Participants } \\
\text { were } \\
\text { recruited } \\
\text { through } \\
\text { social media } \\
\text { and through } \\
\text { national } \\
\text { media } \\
\text { outlets (BBC, } \\
26 \text { March } \\
\text { 2020) and by } \\
\text { distributing } \\
\text { an invitation } \\
\text { to } \\
\text { participate } \\
\text { through } \\
\text { existing } \\
\text { researcher } \\
\text { networks }\end{array}$ & $\begin{array}{l}\text { COVID-19 } \\
\text { pandemic }\end{array}$ & Online survey & $\begin{array}{l}\text { Multivariable } \\
\text { logistic } \\
\text { regression, } \\
\text { chi-squared } \\
\text { tests for } \\
\text { categorical } \\
\text { variables and } \\
t \text {-tests for } \\
\text { continuous } \\
\text { variables- } \\
\text { analysis of } \\
\text { variance } \\
\text { (ANOVA), } \\
\text { Cramer's V, } \\
\text { Cohen's d, } \\
\text { regression } \\
\text { analysis, and } \\
\text { logistic } \\
\text { regression } \\
\text { analysis }\end{array}$ & $\begin{array}{l}\text { A total of } 39.9 \% \text { of the sample } \\
\text { reported engaging in sexual } \\
\text { activity at least once per week } \\
\text { on average, was classified as } \\
\text { sexually active during } \\
\text { lockdown. The mean number } \\
\text { of sexual activities was } 1.75 \text { in } \\
\text { the overall population, and was } \\
\text { significantly higher in men } \\
\text { than women. The prevalence of } \\
\text { sexual activity significantly } \\
\text { increased from } 33.5 \% \text { in people } \\
\text { who were self-isolated for } \\
0-5 \text { days to } 47.0 \% \text { in those who } \\
\text { were self-isolated for } 11 \text { days. } \\
\text { Adults with sexual activity } \\
\text { were mostly male and of a } \\
\text { younger age; married/in a } \\
\text { domestic relationship, } \\
\text { employed, having high annual } \\
\text { household income, and } \\
\text { consuming alcohol, while the } \\
\text { number of chronic physical } \\
\text { conditions was significantly } \\
\text { lower in the sexually active } \\
\text { than in the non-sexually active } \\
\text { group. }\end{array}$ \\
\hline $\begin{array}{l}\text { Nelson K.M. } \\
\text { et al. (2020) }\end{array}$ & US & English & $\begin{array}{l}27 \text { March } \\
2020 \text { to } 8 \\
\text { May } 2020\end{array}$ & $\begin{array}{l}\text { Mixed } \\
\text { method }\end{array}$ & $\begin{array}{l}\text { Adolescents } \\
\text { Sexual } \\
\text { Minority } \\
\text { Males } \\
\text { (ASMM) }\end{array}$ & 151 & $14-17$ & $\begin{array}{l}\text { Online } \\
\text { survey }\end{array}$ & $\begin{array}{l}\text { COVID-19 } \\
\text { pandemic }\end{array}$ & $\begin{array}{l}\text { Demographics, } \\
\text { questions concerning } \\
\text { sexual preferences, } \\
\text { and sexual behavior }\end{array}$ & $\begin{array}{l}\text { Fisher's exact } \\
\text { and } t \text {-tests, } \\
\text { framework } \\
\text { matrix analysis }\end{array}$ & $\begin{array}{l}\text { The participants reported that } \\
\text { they saw their sexual partners } \\
\text { less often, while watching } \\
\text { pornography and masturbating } \\
\text { was more often. Sexting and } \\
\text { messaging through hook-up } \\
\text { apps and websites were used } \\
\text { more frequent during the last } \\
\text { three months. } \\
\text { Social distancing made } \\
\text { physical connection more } \\
\text { difficult and an increase in the } \\
\text { use of virtual means of } \\
\text { communication was reported, } \\
\text { such as video chatting. }\end{array}$ \\
\hline
\end{tabular}


Table 1. Cont.

\begin{tabular}{|c|c|c|c|c|c|c|c|c|c|c|c|c|}
\hline $\begin{array}{l}\text { First Author } \\
\text { (Year) }\end{array}$ & Country & Language & $\begin{array}{l}\text { Study } \\
\text { Period }\end{array}$ & $\begin{array}{c}\text { Study } \\
\text { Design }\end{array}$ & Sample & Sample Size & $\begin{array}{c}\text { Age } \\
\text { Range }\end{array}$ & $\begin{array}{l}\text { Selection of } \\
\text { Sample }\end{array}$ & $\begin{array}{c}\text { Ascertainment } \\
\text { and/or } \\
\text { Association } \\
\text { with the } \\
\text { COVID-19 } \\
\text { Pandemic }\end{array}$ & $\begin{array}{c}\text { Outcomes, } \\
\text { Way/Questionnaires } \\
\text { They Were Measured }\end{array}$ & $\begin{array}{l}\text { Statistical } \\
\text { Analysis }\end{array}$ & Main Findings \\
\hline $\begin{array}{l}\text { Holloway I.W. } \\
\text { et al. (2021) }\end{array}$ & $\begin{array}{l}\text { Multiple } \\
\text { coun- } \\
\text { tries }\end{array}$ & English & $\begin{array}{l}\text { 16 April } \\
2020 \text { to } 24 \\
\text { May } 2020\end{array}$ & $\begin{array}{c}\text { Cross- } \\
\text { sectional }\end{array}$ & $\begin{array}{c}\text { Gay, } \\
\text { Bisexual and } \\
\text { Other Men } \\
\text { Who Have } \\
\text { Sex With } \\
\text { Men } \\
\text { (GBMSM) }\end{array}$ & 10079 & $18-35$ & $\begin{array}{l}\text { Online } \\
\text { survey via } \\
\text { Hornet }\end{array}$ & $\begin{array}{l}\text { COVID-19 } \\
\text { pandemic }\end{array}$ & $\begin{array}{l}\text { 58-question online } \\
\text { survey } \\
\text { regarding the impact } \\
\text { of COVID-19 on a } \\
\text { variety of health and } \\
\text { mental health } \\
\text { outcomes }\end{array}$ & $\begin{array}{c}\text { Chi-squared } \\
\text { tests, } \\
\text { multivariable } \\
\text { logit } \\
\text { regressions }\end{array}$ & $\begin{array}{l}\text { GBMSM who practiced } \\
\text { physical distancing were more } \\
\text { anxious (aOR }=1.37) \text {, lonely } \\
\text { (aOR }=1.36) \text {, and reported that } \\
\text { their sex life was impacted } \\
\text { during the pandemic } \\
(\mathrm{aOR}=2.95) \text {, while a small } \\
\text { number reported satisfaction } \\
\text { with their sex life at the time } \\
(\mathrm{aOR}=0.76) \text {. Social media and } \\
\text { technology was more likely to } \\
\text { be used by those who practiced } \\
\text { physical distancing. }\end{array}$ \\
\hline
\end{tabular}




\subsection{Sexual Activity and Behavior}

According to the studies, a general decrease in sexual desire during the COVID-19 pandemic was reported from individuals [12,13,15,16,19-24]. More specifically, in a Chinese study conducted on youth (age range: 15-35 years old), $41 \%$ reported fewer intercourses, while $20 \%$ also reported a decrease in alcohol consumption before or during sex [12]. Men expressed more often a decrease in sexual partners $(53 \%)$ compared to women $(30 \%)$, while sexual satisfaction was reduced in both genders ( $32 \%$ of men and $39 \%$ of women), according to another Chinese study, in which the majority of the sample was 15-30 years old [13]. Gender and age differences could predict sexual dissatisfaction, along with COVID-19 potential infection during contact and occurrence of depressive symptoms, as stated by an Italian study by Cocci A et al. (mean age 21 years old) [20]. Following the above, another study in Italy (age range 18-40 years old (33.4\% were 18-30 years)) presented a decrease in sexual intercourse by individuals during quarantine, mainly due to a lack of privacy and psychological stimuli [26].

According to Luetke et al., in the US, during home isolation and social distancing, conflicts were inevitable, leading to lower levels of sexual satisfaction mostly in men (a significant percentage (36.7\%) in ages 18-39) [16]. Loneliness and depressive symptoms were associated with fewer sexual intercourses and bonding behaviors between partners in the US study by Hensel et al. (38.3\% participants of ages 18-39) [15]. According to the Chinese study by Jianjun et al., women reported higher scores in the Sexual Compulsivity Scale (SCS) than men, while older counterparts also presented higher scores in SCS from younger ones (age range between 17 and 24 years), and individuals who live in urban areas reported lower scores in SCS than those living in rural areas [14]. The study by Fuchs et al. in Polish women (mean age: 25.1 years) reported a decrease in Female Sexual Function Index (FSFI) scores that was associated with the lack of work before and during the COVID-19 pandemic [23].

On the other hand, a small increase in sexual desire was expressed from men and women in China, Turkey, and France [13,22,24]. Masturbation was a preferable mean of satisfaction, through pornographic content during quarantine [12,20,22,25]. People who self-isolated from $0-5$ days increased their sexual activity (33.5\%) more than people who self-isolated for 11 days or more (47\%) (32.4\% were $18-34$ years) according to a UK study. [19]. Higher knowledge of COVID-19 consequences was associated with more stable sexual behaviors among partners (a significant percentage (38.3\%) in ages 18-39) [15]. According to the above, a significant number of individuals in an Italian study did not report a reduction in their sexual desire $(71.3 \%$, a large proportion of the sample being $18-30$ years) [26].

Concerning couples, a decrease in sexual desire was reported in $10.4 \%$ of females and $9 \%$ of males in a French study (the majority of the sample were 15-30 years old) [22] while a Chinese study reported fewer instances of sexual intercourse by both genders (which also had a majority of the sample in the 15-30-year age group) [13]. Having children under five led to greater instances of sexual release, while having older children (elementary aged) lead to a decrease in those behaviors, as evidenced by Hensel et al. in the USA [15]. Although, many couples reported no difference in their sexual activities in general, in an Italian cross-sectional study ( $61.5 \%$ were under 34 years old), $12.1 \%$ of men and $18.7 \%$ of women stated that there was an increase in their sexual desire [21].

The use of technology, including dating or hooking-up apps, decreased, as evidenced in a study on 15-40+-year-old US men having sex with men [17]. Those apps were used by young people, in order to stay in touch, but not in face-to-face interactions. Thus, the opportunities for sexual intercourse were limited. The reduction of sexual activity among young people (under 17 and over 18 years old), could be reflected in the reduction in demand for sexual health services (SHS) as stated by Thomson-Glover et al. in the UK [18]. Findings support the above data were also presented in a study including Adolescents Sexual Minority Men (ASMM) (14-17 years old), who used social media and virtual means of communications to stay in touch with their sexual partners [25]. In addition, in the 
same context, in a study including Gay, Bisexual, and Other Men Who Have Sex With Men (GBMSM) (age range 18-35 years old), the use of social media for communication was reported, while an impact on sexual life during the ongoing COVID-19 pandemic was expressed [27].

\subsection{Risk of Bias}

The majority of studies were cross-sectional $(n=10)$ and six of them scored high (either 9 or 8 ) in the Newcastle-Ottawa scale. In some cases, the selection of the sample was detailed, but in six of them, the non-responder rate was not justified. The ascertainment of the exposure was implemented through online questionnaires, due to COVID-19 restrictions, and thus were not always validated. Although the control of confounders was performed through appropriate statistical analysis, the outcome was mainly assessed through self-report questionnaires and could not be totally reliable. Furthermore, the five cohort studies provided good $(n=2)$ and fair quality $(n=3)$.

\section{Discussion}

The ongoing COVID-19 pandemic seems to have multiple effects in the sexual life of youth. Adolescents and young adults are the age groups who might be less vulnerable to the virus, but seem to suffer greatly from psychosocial consequences [28]. Social distancing, school closure, and restriction of activities lead to a reduction of any kind of social contact. Additionally, as they were obliged to stay most of the time inside, adolescents and young adults were subjected to increased parental monitoring, which reduced independence, physical interaction with peers, and privacy [29]. Subsequently, according to available reports, a decrease was observed in age groups concerning partnered sex, sexual behaviors, and relationships $[4,29]$. On the other hand, an increase was recorded in online social connections, as $65 \%$ of teenagers used texting or interaction via social media more often than usual [29].

According to our findings, a decrease in sexual desire during the COVID-19 pandemic, concerning fewer intercourses, was reported in general, due to loneliness and depressive symptoms, which were also associated with fewer sexual intercourses [12,13,15,16,19-27]. Sexual partners decreased, especially concerning males, while sexual satisfaction was reduced in both genders (32\% of men and 39\% of women) in the age group of 15-30 years old [13].

Additionally, according to our findings, the knowledge of COVID-19 consequences was associated with more stable sexual behaviors among partners in ages 18-39 [15]. Our study also detected that the use of dating or hooking-up apps decreased during COVID-19 restrictions, according to a study on men having sex with men (age range 15-40 years old) [17]. Nevertheless, online connections seem to be rather important, as they offer options to connect despite the social distancing and stay-at-home orders [29]. Another finding was the reduction in demand for sexual health services, mainly due to fear of infection, among young people [18], possibly associated with problems in accessing condoms, HIV and STI testing, and treatment services, leading to increased rates of sexually transmitted infections and unintended pregnancy among youth [4]. For adolescents and young adults considered to be vulnerable, especially those who are part of the LGBTQ community, who had to face discrimination, violence, and lack of access to health, the COVID-19 pandemic created a more hostile environment for them. Thus, deterioration in their mental health and well-being was noted, including their sex life [30,31].

On the other hand, during COVID-19 and due to the circumstances, methods such as telehealth, home-based sexually transmitted infections screening, and contraceptive delivery were developed [4]. According to the literature, these methods were considered safe and effective and acceptable to youth. Thus, some of them could be adopted afterwards in order to provide better health care concerning the sexual health of these age groups [4]. Inaccessibility of sexual and reproductive healthcare services seems to be one of the main 
causes of increased rates of sexually transmitted infections among adolescents and young adults, a fact that was also highlighted during the pandemic $[4,18]$.

Furthermore, it seems that the COVID-19 pandemic has exposed adolescents and mostly girls to multiplied risks concerning their sexual health, such as sexually transmitted infections including HIV and Human Papilloma Virus, as well as unintended pregnancies [30]. According to reports, an increase was also observed in sexual and gender-based violence, a fact that is probably the result of the difficulty in accessing relevant services such as intervention programs [30]. Thus, the pandemic seems to have additionally highlighted the need for better organization and the development of youth-friendly and easy-to-access sexual and reproductive healthcare services. Concerning the limitations of this study, the COVID-19 pandemic is an ongoing phenomenon, and thus, the impact in the sexual activities of adolescents and young adults need to be further tested. In order to provide a global understanding of relevant effects, an examination of diverse groups should be a priority. Confinement measures and social distancing created a more complex reality, affecting all aspects of social life; the interplay with sexual life should be monitored in a more systematic way. Furthermore, the majority of the studies were cross-sectional, providing no long-term results, while self-reported assessments limited the validity of studies.

\section{Conclusions}

In conclusion, the present paper highlights that sexual activity, an important aspect of adolescents' and young adults' life, was reported to be considerably affected during the ongoing COVID-19 pandemic. The changes observed in sexual activity and relationships could play an important role in forming preventive and educational programs in collaboration with parents, caregivers, teachers, and medical staff, aimed towards good sexual health and well-being.

Author Contributions: Conceptualization, T.P., T.N.S. and A.T. (Artemis Tsitsika); methodology, A.S., C.S., E.P., A.T. (Athanasios Thirios), A.K. and T.N.S.; investigation, A.S., C.S., A.K., and S.T.; writing —original draft preparation, A.S., C.S., E.P., A.T. (Athanasios Thirios), and A.K.; writing-review and editing T.P., T.N.S. and A.T. (Artemis Tsitsika); visualization, A.T. (Athanasios Thirios), T.P. and E.P.; supervision, T.P., A.T. (Artemis Tsitsika) and T.N.S. All authors have read and agreed to the published version of the manuscript.

Funding: This research received no external funding.

Institutional Review Board Statement: Not applicable.

Informed Consent Statement: Not applicable.

Data Availability Statement: Data is contained within the article.

Conflicts of Interest: The authors declare no conflict of interest.

\section{References}

1. Rothe, C.; Schunk, M.; Sothmann, P.; Bretzel, G.; Froeschl, G.; Wallrauch, C.; Zimmer, T.; Thiel, V.; Janke, C.; Guggemos, W.; et al. Transmission of 2019-nCoV Infection from an Asymptomatic Contact in Germany. N. Engl. J. Med. 2020, 382, 970-971. [CrossRef] [PubMed]

2. Gagliano, A.; Villani, P.G.; Co', F.M.; Manelli, A.; Paglia, S.; Bisagni, P.A.G.; Perotti, G.; Storti, E.; Lombardo, M. COVID-19 Epidemic in the Middle Province of Northern Italy: Impact, Logistics, and Strategy in the First Line Hospital. Disaster Med. Public Health Prep. 2020, 14, 372-376. [CrossRef] [PubMed]

3. Adalja, A.A.; Toner, E.; Inglesby, T.V. Priorities for the US Health Community Responding to COVID-19. JAMA 2020, 323, 1343. [CrossRef] [PubMed]

4. Mmeje, O.O.; Coleman, J.S.; Chang, T. Unintended Consequences of the COVID-19 Pandemic on the Sexual and Reproductive Health of Youth. J. Adolesc. Health 2020, 67, 326-327. [CrossRef] [PubMed]

5. Lindberg, L.D.; Bell, D.L.; Kantor, L.M. The Sexual and Reproductive Health of Adolescents and Young Adults during the COVID-19 Pandemic. Perspect. Sex. Reprod. Health 2020, 52, 75-79. [CrossRef] 
6. $\quad$ Emans, S.J.; Ford, C.A.; Irwin, C.E.; Richardson, L.P.; Sherer, S.; Sieving, R.E.; Simpson, T. Early COVID-19 Impact on Adolescent Health and Medicine Programs in the United States: LEAH Program Leadership Reflections. J. Adolesc. Health 2020, 67, 11-15. [CrossRef]

7. UNFPA. International Technical Guidance on Sexuality Education-An Evidence-Informed Approach; UNESCO, UN-AIDS, UNFPA, UNICEF, UN Women and WHO: New York, NY, USA, 2018.

8. Ott, M.A.; Bernard, C.; Wilkinson, T.A.; Edmonds, B.T. Clinician Perspectives on Ethics and COVID-19: Minding the Gap in Sexual and Reproductive Health. Perspect. Sex. Reprod. Health 2020, 52, 145-149. [CrossRef]

9. Perissini, A.L.; Spessoto, L.C.F.; Junior, F.N.F. Does online pornography influence the sexuality of adolescents during COVID-19? Rev. Assoc. Med. Bras. 2020, 66, 564-565. [CrossRef]

10. Modesti, P.A.; Reboldi, G.; Cappuccio, F.P.; Agyemang, C.; Remuzzi, G.; Rapi, S.; Perruolo, E.; Parati, G.; ESH Working Group on CV Risk in Low Resource Settings. Panethnic Differences in Blood Pressure in Europe: A Systematic Review and Meta-Analysis. PLoS ONE 2016, 11, e0147601. [CrossRef]

11. Stang, A. Critical evaluation of the Newcastle-Ottawa scale for the assessment of the quality of nonrandomized studies in meta-analyses. Eur. J. Epidemiol. 2010, 25, 603-605. [CrossRef]

12. Li, G.; Tang, D.; Song, B.; Wang, C.; Qunshan, S.; Xu, C.; Geng, H.; Wu, H.; He, X.; Cao, Y. Impact of the COVID-19 Pandemic on Partner Relationships and Sexual and Reproductive Health: Cross-Sectional, Online Survey Study. J. Med. Internet Res. 2020, 22, e20961. [CrossRef] [PubMed]

13. Li, W.; Li, G.; Xin, C.; Wang, Y.; Yang, S. Challenges in the Practice of Sexual Medicine in the Time of COVID-19 in China. J. Sex. Med. 2020, 17, 1225-1228. [CrossRef] [PubMed]

14. Deng, J.; Li, T.; Wang, J.; Teng, L. The Effect of COVID-19 Stress on Sexual Compulsivity Symptom: The Mediating Role of Perceived Social Support. Res. Sq. 2020. [CrossRef]

15. Hensel, D.J.; Rosenberg, M.; Luetke, M.; Fu, T.; Herbenick, D. Changes in Solo and Partnered Sexual Behaviors during the COVID-19 Pandemic: Findings from a U.S. Probability Survey. medRxiv 2020. [CrossRef]

16. Luetke, M.; Hensel, D.; Herbenick, D.; Rosenberg, M. Romantic Relationship Conflict Due to the COVID-19 Pandemic and Changes in Intimate and Sexual Behaviors in a Nationally Representative Sample of American Adults. J. Sex Marital. Ther. 2020, 46, 747-762. [CrossRef]

17. Sanchez, T.H.; Zlotorzynska, M.; Rai, M.; Baral, S.D. Characterizing the Impact of COVID-19 on Men Who Have Sex with Men across the United States in April, 2020. AIDS Behav. 2020, 24, 2024-2032. [CrossRef]

18. Thomson-Glover, R.; Hamlett, H.; Weston, D.; Ashby, J. Coronavirus (COVID-19) and young people's sexual health. Sex. Transm. Infect. 2020, 96, 473-474. [CrossRef]

19. Jacob, L.; Smith, L.; Butler, L.; Barnett, Y.; Grabovac, I.; McDermott, D.; Armstrong, N.; Yakkundi, A.; Tully, M.A. Challenges in the Practice of Sexual Medicine in the Time of COVID-19 in the United Kingdom. J. Sex. Med. 2020, 17, 1229-1236. [CrossRef]

20. Cocci, A.; Giunti, D.; Tonioni, C.; Cacciamani, G.; Tellini, R.; Polloni, G.; Cito, G.; Presicce, F.; Di Mauro, M.; Minervini, A.; et al. Love at the time of the Covid-19 pandemic: Preliminary results of an online survey conducted during the quarantine in Italy. Int. J. Impot. Res. 2020, 32, 556-557. [CrossRef]

21. Panzeri, M.; Ferrucci, R.; Cozza, A.; Fontanesi, L. Changes in Sexuality and Quality of Couple Relationship during the COVID-19 Lockdown. Front. Psychol. 2020, 11, 565823. [CrossRef]

22. Landry, S.; Chartogne, M.; Landry, A. The impacts of COVID-19 lockdown on sexuality. Sexologies 2020, 29, 173-180. [CrossRef]

23. Fuchs, A.; Matonóg, A.; Pilarska, J.; Sieradzka, P.; Szul, M.; Czuba, B.; Drosdzol-Cop, A. The Impact of COVID-19 on Female Sexual Health. Int. J. Environ. Res. Public Health 2020, 17, 7152. [CrossRef]

24. Yuksel, B.; Ozgor, F. Effect of the COVID-19 pandemic on female sexual behavior. Int. J. Gynecol. Obstet. 2020, 150, 98-102. [CrossRef]

25. Nelson, K.M.; Gordon, A.R.; John, S.A.; Stout, C.D.; Macapagal, K. "Physical Sex Is Over for Now": Impact of COVID-19 on the Well-Being and Sexual Health of Adolescent Sexual Minority Males in the U.S. J. Adolesc. Health 2020, 67, 756-762. [CrossRef]

26. Cito, G.; Micelli, E.; Cocci, A.; Polloni, G.; Russo, G.I.; Coccia, M.E.; Simoncini, T.; Carini, M.; Minervini, A.; Natali, A. The Impact of the COVID-19 Quarantine on Sexual Life in Italy. Urology 2021, 147, 37-42. [CrossRef] [PubMed]

27. Holloway, I.W.; Garner, A.; Tan, D.; Ochoa, A.M.; Santos, G.M.; Howell, S. Associations Between Physical Distancing and Mental Health, Sexual Health and Technology Use Among Gay, Bisexual and Other Men Who Have Sex With Men during the COVID-19 Pandemic. J. Homosex. 2021, 68, 692-708. [CrossRef] [PubMed]

28. Stavridou, A.; Bsc, A.S.; Panagouli, E.; Mesiris, G.; Thirios, A.; Mougiakos, T.; Troupis, T.; Psaltopoulou, T.; Tsolia, M.; Sergentanis, T.N.; et al. Psychosocial consequences of COVID-19 in children, adolescents and young adults: A systematic review. Psychiatry Clin. Neurosci. 2020, 74, 615-616. [CrossRef] [PubMed]

29. Assari, S.; Caldwell, C.H.; Bazargan, M. Parental Educational Attainment and Black-White Adolescents' Achievement Gap: Blacks' Diminished Returns. Open J. Soc. Sci. 2020, 08, 282-297. [CrossRef]

30. Murewanhema, G. Adolescent girls, a forgotten population in resource-limited settings in the COVID-19 pandemic: Implications for sexual and reproductive health outcomes. Pan Afr. Med. J. 2020, 37. [CrossRef]

31. Salerno, J.P.; Devadas, J.; Pease, M.; Nketia, B.; Fish, J.N. Sexual and Gender Minority Stress Amid the COVID-19 Pandemic: Implications for LGBTQ Young Persons' Mental Health and Well-Being. Public Health Rep. 2020, 135, 721-727. [CrossRef] [PubMed] 